\title{
Hiperplasia suprarrenal no clásica, características de laboratorio y respuesta al tratamiento. Estudio de cohorte
}

\author{
Non-classic adrenal hyperplasia, laboratory characteristics and treatment \\ response. Cohort study
}

\author{
Abreu Lomba A, ${ }^{1}$ González Bermúdez C, ${ }^{2}$ Salazar LC, ${ }^{2}$ Bolena Muriel A, ${ }^{2}$ Gómez MC, ${ }^{3}$ \\ Hernández-Carrillo M. ${ }^{4}$
}

${ }^{1}$ Departamento de Endocrinología, Centro Médico Imbanaco. Docente, Universidad libre, Residencia en Ginecología y Obstetricia y Medicina Interna. Cali, Colombia.

${ }^{2}$ Residente Ginecología y Obstetricia, Universidad Libre. Cali, Colombia.

${ }^{3}$ Coordinador posgrado en Ginecología y Obstetricia, Universidad Libre. Cali, Colombia.

${ }^{4}$ Estadístico y Magíster en Epidemiología. Docente, Universidad Libre, Grupo GISAP. Cali, Colombia.

Autor de correspondencia: Alin Abreu Lomba

Correo electrónico: alin.lomba@gmail.com

Fecha de recepción: 25/06/2020

Fecha de aceptación: 11/02/2021

\section{Resumen}

Objetivo: comparar las características de laboratorio y la respuesta al tratamiento en una cohorte de pacientes con hiperplasia suprarrenal congénita no clásica seguidas durante 12 meses.

Métodos: se realizó un estudio en una cohorte final de 38 pacientes con hiperplasia suprarrenal no clásica evaluadas entre enero de 2006 hasta diciembre de 2016 y que tuvieron seguimiento a 1 año. Las características de la población de estudio y la respuesta de 17-hidroxiprogesterona (17-OH progesterona), testosterona y dehidroepiandrosterona sulfato (DHEA-S) se informan a los 6 y 12 meses después del tratamiento con dexametasona y etinilestradiol/acetato de ciproterona. Se realizó una regresión lineal múltiple para explicar el cambio de las variables de resultado (17- $\mathrm{OH}$ progesterona, testosterona y DHEA-S), ajustado de acuerdo con el índice de masa corporal (IMC) y la edad como covariables.

Resultados: la edad promedio de los participantes fue de 25 años, el IMC promedio fue de $28,3 \mathrm{~kg} / \mathrm{m}^{2}$. Las característi- cas clínicas más prevalentes fueron el acné $(52,6 \%$ ) y la oligomenorrea (55\%). El hirsutismo se presentó en todos los casos en algún grado (moderado: 31,6 \% y grave: 39,5 \%, según la escala de Ferriman y Gallwey). El cambio en los niveles de cada una de las hormonas evidenció una disminución significativa de la mediana del rango intercuartílico de 17-0H progesterona, testosterona y DHEA-S, a los 6 y 12 meses $(p<0,05)$. En la regresión lineal múltiple, los niveles de testosterona presentaron una variación de acuerdo con el $\operatorname{IMC}(p=0,04)$. En el presente estudio, la terapia farmacológica con dexametasona se aplicó a diferentes dosis y todas las pacientes recibieron anticonceptivos con una respuesta clínica y paraclínica adecuada al año de seguimiento.

Conclusión: este estudio sugiere que la combinación de etinilestradiol más acetato de ciproterona con dosis variables de dexametasona disminuye el perfil androgénico en las pacientes con hiperplasia suprarrenal no clásica seguidas durante un año.

Palabras clave: hiperplasia suprarrenal congénita, esteroide 21-hidroxilasa, 17-alfa- hidroxiprogesterona, testosterona, deshidroepiandrosterona, hiperandrogenismo, hormona adrenocorticotrópica.

\section{Abstract}

Objective: To compare the laboratory characteristics and response to treatment in a cohort of patients with non-classical congenital adrenal hyperplasia followed during 12 months.

Methods: A study was conducted in a cohort of 38 patients with non-classical adrenal hyperplasia from January 2006 to December 2016. The characteristics of the study population and the response of 17-hydroxyprogesterone (17-OH progesterone), testosterone, and dehydroepiandrosterone sulfate (DHEA-S) are reported 6 and 12 months after treatment with 
dexamethasone, and ethinyl estradiol/cyproterone acetate. A multiple linear regression analysis was performed in order to explain the change of the outcome variables (17-OH progesterone, testosterone, and DHEA-S), adjusted according to body mass index (BMI) and age as covariates.

Results: The average age of the participants was 25 years and average BMI was $28.3 \mathrm{~kg} / \mathrm{m}^{2}$. The most prevalent clinical features were acne (52.6\%) and oligomenorrhea (55\%). Hirsutism occurred to some degree in all cases (31.6\% moderate and 39.5\% severe, according to the Ferriman-Gallwey scale). The change in the levels of each hormone reflected a significant decrease in the median and interquartile range levels of 17-OH progesterone, testosterone and DHEA-S, at 6 and 12 months $(p<0.05)$. In the multiple linear regression, the levels of testosterone showed variation according to BMI $(p=0.04)$. In this study, pharmacological therapy with dexamethasone was applied at different doses and all patients were given contraceptives, with adequate clinical and paraclinical response at 1 year of follow-up.

Conclusion: This study suggests that the combination of cyproterone plus ethinyl estradiol with variable doses of dexamethasone decreases the androgenic profile in patients with non-classic adrenal hyperplasia followed over a 1-year period.

Keywords: Adrenal Hyperplasia, Congenital; Steroid21-Hydroxylase; 17-alpha-Hydroxyprogesterone; Testosterone; Dehydroepiandrosterone; Hyperandrogenism; Adrenocorticotropic Hormone.

\section{Introducción}

La hiperplasia suprarrenal congénita (HSC-NC) es un trastorno autosómico recesivo que se caracteriza por presentar un defecto enzimático en algún paso de la biosíntesis de glucocorticoides y mineralocorticoides en las glándulas suprarrenales (cortisol, aldosterona), asociado con una sobreestimulación de andrógenos (1). La forma más frecuente es la deficiencia de la enzima 21-hidroxilasa, que representa el $95 \%$ de los casos de hiperplasia suprarrenal congénita (2). Los 2 fenotipos más frecuentes de la enfermedad incluyen la forma clásica, que se subdivide en perdedora de sal y virilizante simple; y la forma de inicio tardío o no clásica, la cual es el centro del presente estudio $(1,3)$.

El fenotipo no clásico de hiperplasia suprarrenal congénita tiene una incidencia de $1 / 1000$ casos por año, con una frecuencia del $0,1 \%$ al $1 \%$. Puede cursar asintomática o estar asociada con signos indicativos de exceso de andrógenos durante la niñez o en el período de la adolescencia y adultez $(1,4,5)$. Durante la niñez, la principal manifestación clínica es la pubarquia precoz, la cual se caracteriza por la aparición de vello púbico, vello axilar y olor apocrino antes de los 8 años en las niñas y antes de los 9 años en los niños; otras manifestaciones son talla alta, clitoromegalia en la niña o crecimiento peneano con testículos pequeños en el niño $(6,7)$. Durante la pubertad, las manifestaciones clínicas más frecuentes son el hirsutismo, amenorrea primaria u oligomenorrea, el acné (8), además de un rápido crecimiento durante la pubertad con talla baja final (7). En la vida adulta, en las mujeres la clínica puede ser similar a un síndrome de ovario poliquístico, un hiperandrogenismo grave secundario a un tumor productor de andrógenos o infertilidad hasta en el $15 \%$ al $30 \%$ de ellas. Los pacientes heterocigotos portadores de una mutación grave pueden tener síntomas de hiperandrogenismo más marcados (9). Por su parte, en el hombre adulto el cuadro clínico es más sutil, pudiéndose encontrar alopecia, oligospermia e infertilidad (10).

El diagnóstico de hiperplasia suprarrenal congénita no clásica se basa en la determinación de las concentraciones plasmáticas de 17-OH progesterona basales y estimuladas con corticotropina (ACTH) (3), y se confirma con el estudio molecular (11). La determinación de los niveles de mineralocorticoides, actividad de renina plasmática y cortisol no ayudan en la práctica clínica en el diagnóstico ni en la evaluación del cuadro a largo plazo.

La necesidad de tratamiento es aún controvertida, pero este se fundamenta en suprimir el exceso de andrógenos que se produce por el déficit enzimático de 21-hidroxilasa, lo cual se controla a través de glucocorticoides tipo hidrocortisona y análogos como prednisona y dexametasona; según el grado de hirsutismo de la paciente, el uso de un antiandrogénico está avalado por la Sociedad de Endocrinología (12). No se indica tratamiento a pacientes asintomáticos $(8,12)$. La adrenalectomía como manejo quirúrgico podría ser una alternativa para suprimir la síntesis de andrógenos, pero debido a los resultados de riesgo quirúrgico, a la baja o nula respuesta al estrés secundario a la deficiencia de epinefrina y al riesgo de desarrollar el síndrome de Nelson (tumor hipofisario productor de ACTH), este manejo debe considerarse como último recurso en pacientes con falla al manejo médico y con infertilidad (13, 14). Otros procedimientos reportados en la literatura son la genitoplastia a temprana edad, pero se ha vuelto cada vez más polémica debido a la falta de datos sobre el resultado funcional a largo plazo (15).

Ante la ausencia de datos epidemiológicos en nuestro entorno, se decidió identificar y monitorizar el comportamiento hormonal y la respuesta al tratamiento en una sola cohorte de pacientes con diagnóstico clínico y paraclínico de hiperplasia suprarrenal no clásica, que asistieron a una clínica de alta complejidad en la ciudad de Cali.

\section{Materiales y métodos}

Se realizó un estudio en una cohorte de 41 pacientes que asistieron al servicio de endocrinología del Centro Médico Imbanaco, institución de salud de alta complejidad ubicada en la ciudad de Cali, Colombia, entre enero de 2006 y diciembre de 2016. Se excluyeron 3 pacientes de la cohorte por laboratorios incompletos, pérdida del seguimiento antes de los 6 meses y falta de adherencia al tratamiento, por lo que la cohorte final 
fue de 38 pacientes con diagnóstico de hiperplasia suprarrenal congénita no clásica, quienes tuvieron seguimiento durante al menos un año en el servicio de endocrinología.

El diagnóstico de hiperplasia suprarrenal congénita no clásica se realizó inicialmente debido a una sospecha clínica y su posterior confirmación paraclínica con niveles plasmáticos de 17-OH progesterona basal, y después a una estimulación con ACTH a una dosis de $250 \mu \mathrm{g}$; no se realizó un diagnóstico definitivo molecular debido a las limitaciones técnicas y económicas de las pacientes. Las pruebas hormonales se analizaron utilizando la técnica de inmunoensayo en el laboratorio de la institución, con controles de calidad internos y externos. Se tomaron los siguientes valores normales de $17-0 \mathrm{H}$ progesterona en fase folicular: 0,1-0,8 ng/mL, fase lútea: 0,6-2,3 ng/mL, pos-ACTH $\leq$ 3,2 ng/mL, testosterona: 6-86 ng/dL y DHEA-S: 30-260 $\mu \mathrm{g} / \mathrm{dL}$.
El seguimiento total de cada paciente fue de 1 año después del diagnóstico y comienzo del tratamiento (Figura 1).

Las características clínicas y los niveles de 17-0H progesterona, testosterona y DHEA-S se describen a los 6 y 12 meses después del tratamiento con dexametasona y etinilestradiol $35 \mathrm{mg}$ /acetato de ciproterona $2 \mathrm{mg}$. La evaluación del grado de hirsutismo fue realizada a través de la escala de Ferriman y Gallwey (16).

Las dosis recomendadas de corticoides varían y se deben basar según la variabilidad clínica, genotipo, grado de severidad enzimático, a lo cual estudios recomiendan individualizar la dosis; algunos artículos consideran dexametasona a dosis entre 0,25 a 0,5 mg/día $(17,18)$. En el actual estudio, la dosis utilizada de dexametasona se ajustó de acuerdo con la variación bioquímica de cada paciente según los niveles de 17-OH progesterona

Figura 1. Diagrama de flujo.

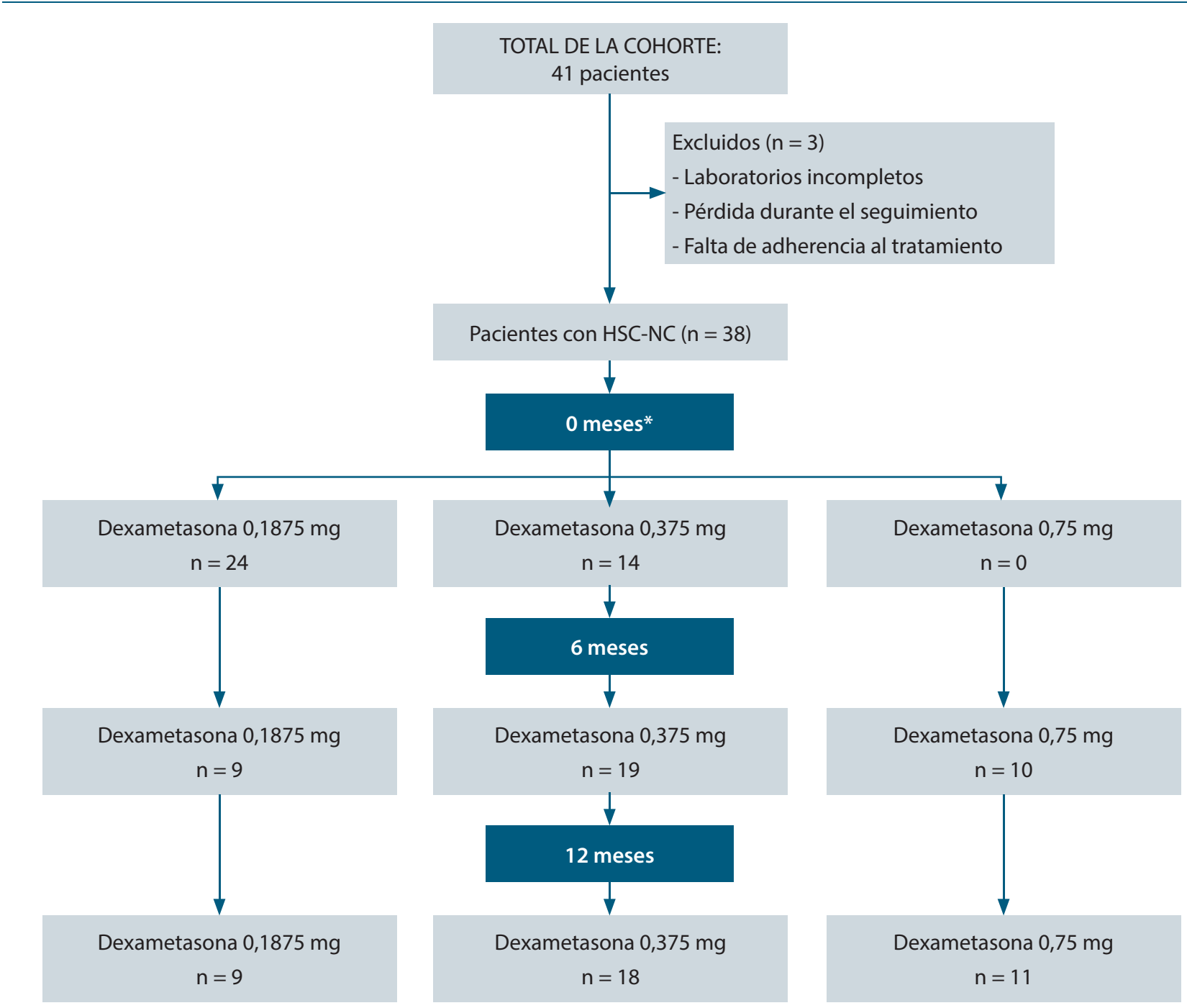

*Se administró etinilestradiol más acetato de ciproterona (35 mg/2 mg) a dosis fijas en todos los pacientes. 
basal. Se tomó como punto de corte el valor de $2 \mathrm{ng} / \mathrm{mL}$, y el aumento de dosis se realizó de forma gradual de la siguiente manera: en el momento cero (0), una dosis mínima de 0,1875 mg/día (cuarto de tableta) y una dosis máxima de 0,375 mg/día (media tableta); y después de 6 y 12 meses de tratamiento se indicó una dosis mínima de 0,1875 mg/día (cuarto de tableta) y una dosis máxima de 0,75 mg/día (1 tableta). El antiandrógeno manejado fue el etinilestradiol $35 \mathrm{mg} /$ acetato de ciproterona $2 \mathrm{mg}$ a una dosis fija para todas las pacientes.

Se realizó el consentimiento informado a cada una de las pacientes incluidas en el estudio y el protocolo del estudio fue aprobado por el comité de ética de la institución.

En cuanto al análisis estadístico, se realizó un análisis univariado, cuyas variables cuantitativas se presentan mediante medias y medianas, y la dispersión, como desviación estándar (DE) o rango intercuartílico (RIC), respectivamente, de acuerdo con el cumplimiento de los supuestos de normalidad; las variables cualitativas se expresaron en frecuencias absolutas y relativas. Para comparar los valores de los laboratorios entre la línea de base que fue 0 meses, luego a los 6 y 12 meses, se realizó un análisis de varianza no paramétrica con contraste de hipótesis utilizando la prueba de Kruskal-Wallis y se emplearon diagramas de cajas para describir estas diferencias. Se realizó el análisis mediante la regresión lineal múltiple para ajustar la magnitud del cambio entre el mes 0 y el mes 12 de seguimiento en los niveles de 17-0H progesterona, testosterona y DHEA-S, por índice de masa corporal (IMC), dosis de corticosteroides y edad como covariables. El modelo final se determinó teniendo en cuenta el diagnóstico de la regresión y la prueba de bondad de ajuste ( $R$-squared). Se consideró un valor $p<0,05$ para establecer diferencias estadísticamente significativas. El procesamiento estadístico se realizó utilizando Excel y el software estadístico Stata, versión 14.

\section{Resultados}

Se completó el seguimiento en 38 de las 41 pacientes incluidas inicialmente (Tabla 1). Las características clínicas más prevalentes fueron acné (52,6\%), oligomenorrea (55 \%) e hirsutismo. El hirsutismo estuvo presente en todos los pacientes y el 39,5 \% de los pacientes tenía un grado grave. El promedio de las pacientes tenía sobrepeso y la tercera parte consultó por amenorrea primaria con hallazgos clínicos de resistencia a la insulina.

El tratamiento con etinilestradiol más acetato de ciproterona (35 mg/2 mg) se mantuvo a una dosis fija durante el período de seguimiento. La dosis de dexametasona se ajustó cada 6 meses de acuerdo con los niveles de 17-0H progesterona basal. Se comenzó con una dosis mínima de 0,1875 mg/ día y una dosis máxima de 0,375 mg/día en el tiempo cero (0), y una dosis mínima de 0,1875 mg/día y una dosis máxima de 0,75 mg/día a los 6 y 12 meses del tratamiento (Figura 2).
Tabla 1. Características sociodemográficas y clínicas de las mujeres con hiperplasia suprarrenal no clásica

\begin{tabular}{l|c}
\hline \multicolumn{1}{c|}{ Características } & Pacientes $(\mathbf{n}=38)$ \\
\hline Edad* & $25,02 \pm 8,9$ \\
\hline Peso $(\mathrm{kg})$ & $72,65 \pm 11,3$ \\
\hline Talla $(\mathrm{cm})$ & $160 \pm 4$ \\
\hline IMC & $28,3 \pm 4,3$ \\
\hline Etnia & \\
Mestizo & $29(76,3 \%)$ \\
Afrocolombiano & $9(23,7 \%)$ \\
\hline Manifestaciones clínicas & \\
Acné & $20(52,6 \%)$ \\
Alopecia & $11(29 \%)$ \\
Oligomenorrea & $21(55 \%)$ \\
Amenorrea primaria & $13(34 \%)$ \\
Amenorrea secundaria & $4(11 \%)$ \\
Acantosis nigricans & $12(32 \%)$ \\
\hline Hirsutismo & $12(31,6 \%)$ \\
Leve & $139,5 \%)$ \\
Moderado & \\
Grave & \\
\hline
\end{tabular}

$*$ Media \pm DE

La respuesta clínica fue adecuada con la dosis intermedia de 0,375 mg a los 6 meses en 19 pacientes. En 11 pacientes fue necesario ajustar la dosis máxima de $0,75 \mathrm{mg}$ a los 12 meses. La dosis más baja de dexametasona se conservó hasta los 12 meses en 9 pacientes.

La mediana del nivel de 17-0H progesterona al inicio del estudio fue de 15,9 ng/mL (RIC: 12-19); a los 6 meses, de 1,65 ng/mL (RIC: 1,4-3,3); y a los 12 meses, de 1,1 ng/mL (RIC: 0,73-1,7) (Figura 3 A). La mediana de los niveles totales de testosterona al inicio del estudio fue de $103 \mathrm{ng} / \mathrm{dL}$ (RIC: 79138); a los 6 meses, de 62 ng/dL (RIC: 50-77); y a los 12 meses, de 48 ng/dL (RIC: 32-59) (Figura 3 B). La mediana de los valores iniciales de DHEA-S fue de $452 \mu \mathrm{g} / \mathrm{dL}$ (RIC: 370-536); a los 6 meses, de $209 \mu \mathrm{g} / \mathrm{dL}$ (RIC: 154-306); y a los 12 meses, de $144 \mu \mathrm{g} / \mathrm{dL}$ (RIC: 105-208) (Figura 3 C). La disminución de los valores de todas las pruebas hormonales fue progresiva y estadísticamente significativa $(p<0,05)$.

Además, se evaluó el cambio en la testosterona, la 17-OH progesterona, la DHEA-S, el IMC ajustado, el aumento de las dosis de corticosteroides y la edad, en el modelo lineal multivariado. En relación con la 17-0H progesterona, el nivel está influenciado por el IMC $(p=0,038)$ independientemente de la 
Figura 2. Porcentaje de pacientes con las diferentes dosis de dexametasona.

Azul oscuro: 0,1875 mg: 1/4 de tableta; gris: 0,375 mg: 1/2 tableta; azul claro: 0,75 mg: 1 tableta.

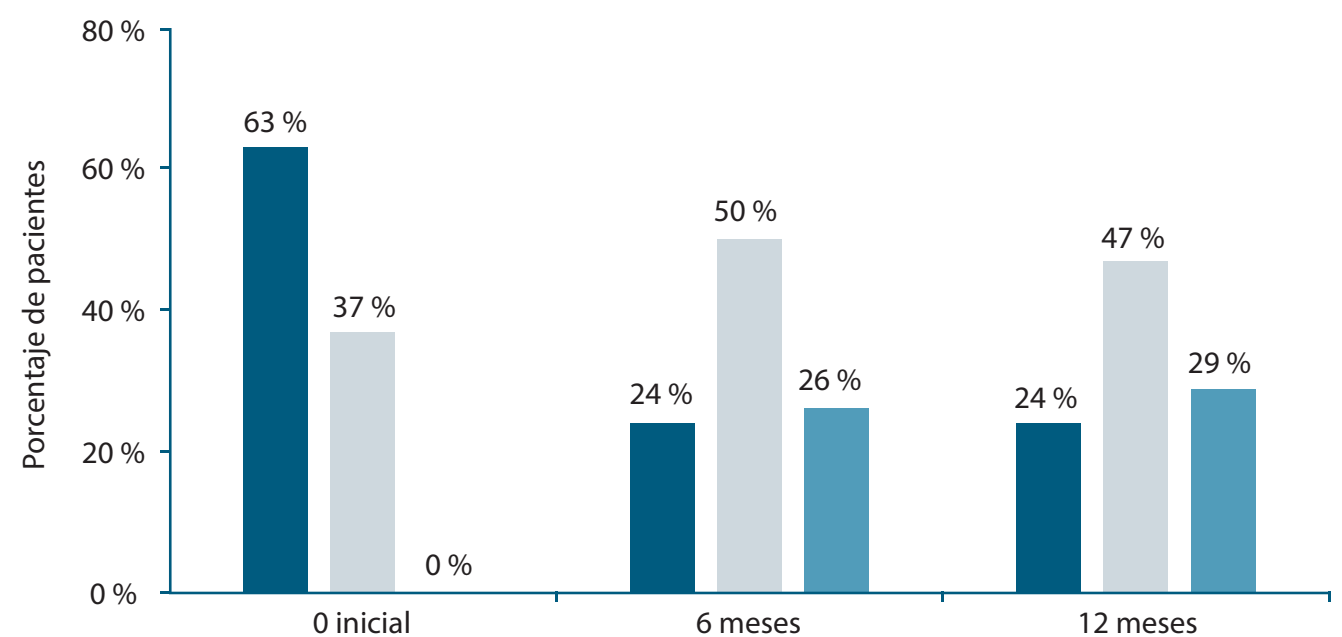

Figura 3. Cambios en los niveles de 17-0H progesterona, DHEA-S y testosterona en el tiempo de seguimiento. Resultado de los paraclínicos en el tiempo inicial, a los 6 meses y a los 12 meses. A. 17-0H progesterona. B. Testosterona total.

C. DHEA-S. Los bordes inferior y superior de cada caja representan el primer y el tercer cuartil, respectivamente; la banda dentro del cuadro representa el valor mediano. En la caja se encuentra el $50 \%$ de los datos, con una $p$ estadísticamente significativa $(p<0,05)$.

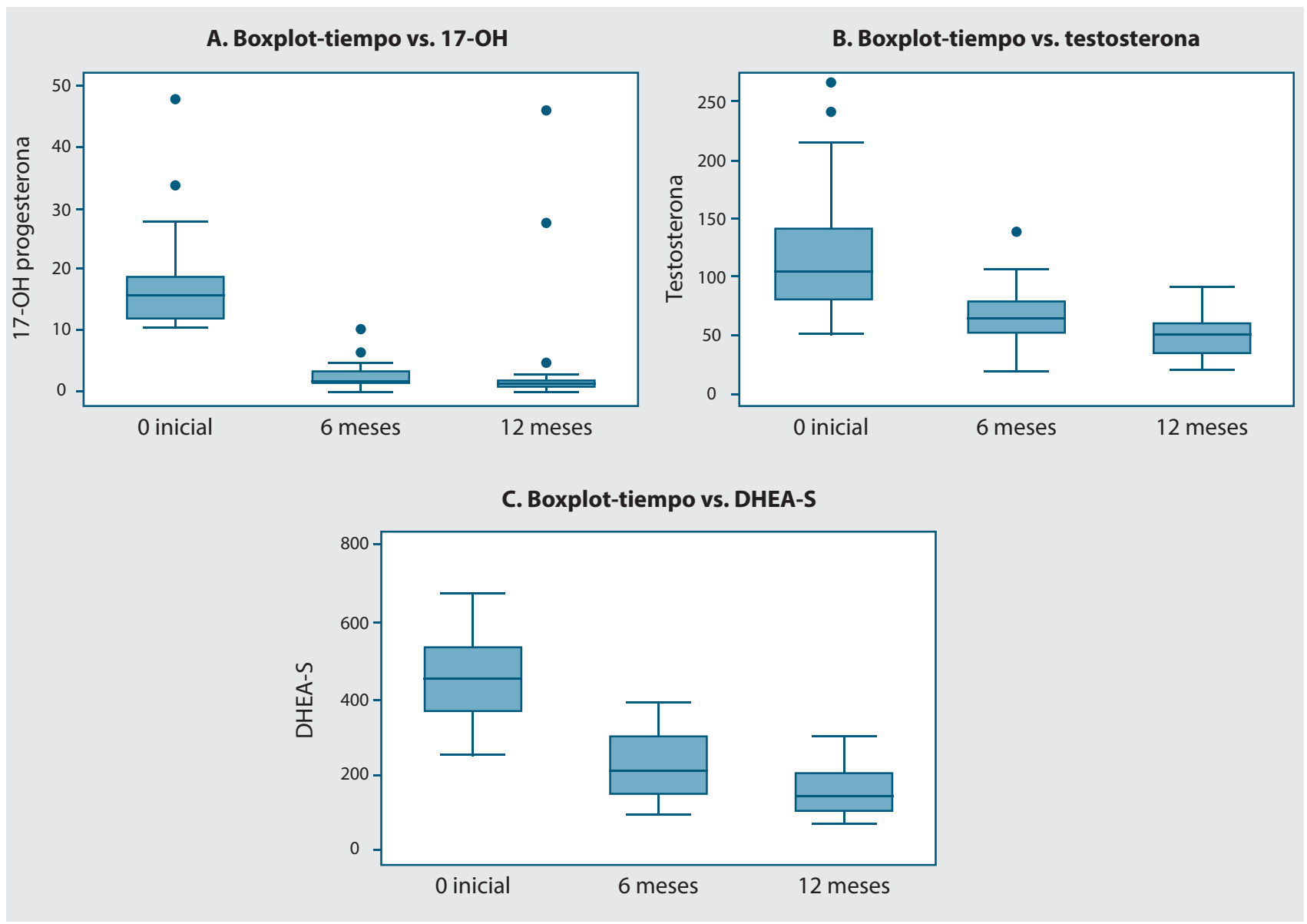


edad y las dosis de corticosteroides. El cambio de testosterona y DHEA-S depende de su nivel basal y no está influenciado por otras covariables (Tabla 2).

\section{Discusión}

La hiperplasia suprarrenal congénita no clásica es una patología autosómica recesiva de baja incidencia, aproximadamente 1/1000 casos por año. Su característica principal es el hiperandrogenismo de origen suprarrenal, que se manifiesta en cualquier etapa de la vida $(1,4)$. La mayoría de los casos se debe a alteraciones del gen CYP21A2, que se encuentra en el cromosoma 6p21.3 (6). Es común que esta patología esté subdiagnosticada porque su presentación clínica es similar a otras patologías, como el síndrome de ovario poliquístico durante la vida adulta $(19,20)$.

El diagnóstico bioquímico de esta patología está relacionado con la deficiencia de la enzima 21-hidroxilasa en el $95 \%$ de los casos y se logra midiendo los niveles de $17-0 \mathrm{H}$ progesterona basal y su posterior estimulación con ACTH; las concentraciones de $17-\mathrm{OH}$ progesterona pos-ACTH $\geq 10 \mathrm{ng} / \mathrm{mL}$ (30 nmol/L) (18) hacen el diagnóstico. Se recomienda que a los pacientes con clínica de hiperandrogenismo y un nivel basal de 17-OH progesterona en la fase folicular del ciclo $>2 \mathrm{ng} / \mathrm{mL}(6 \mathrm{nmol} / \mathrm{L}$; punto de corte, sensibilidad del $100 \%$ [IC $95 \%$ : 59,04-100] y especificidad del $93 \%$ [IC 95 \%: 85,3-97,37]) (21) se les realice la prueba confirmatoria de estimulación con ACTH $(22,23)$.

Una vez realizado el diagnóstico de la enfermedad, el siguiente paso es el tratamiento oportuno y adecuado, considerando la terapia con glucocorticoides como el pilar principal del tratamiento para esta enfermedad $(12,17,18,21)$. No existe un consenso global sobre los regímenes de esteroides porque las indicaciones terapéuticas dependen de varios factores como la edad, los síntomas principales y la potencia del esteroide elegido. En caso de iniciar el tratamiento en niños con pubarquia temprana, El-Maouche y colaboradores (6) recomiendan la hidrocortisona en dosis bajas, generalmente a la mitad de la dosis en la forma clásica de 8-10 mg/m²/día en 2-3 dosis por día; la prednisona y la dexametasona solo se usan una vez que se complete el crecimiento (12). Por su parte, la Sociedad de Endocrinología de Chile recomienda el uso de dexametasona a una dosis de 0,25-0,5 mg en adolescentes y adultos; sin embargo, en la edad adulta la supresión de glucocorticoides aislada rara vez controla el hirsutismo y frecuentemente requiere una terapia antiandrogénica adicional con acetato de ciproterona, clormadinona o espironolactona junto con anticonceptivos orales a base de estrógenos para reducir de manera más efectiva los andrógenos circulantes; este tratamiento también se sugiere en varios ensayos clínicos $(11,15,24)$.

Por una parte, Falhammar y colaboradores compararon la efectividad del acetato de ciproterona frente a la hidrocortisona, con una disminución del hirsutismo en el $54 \%$ en el grupo de ciproterona, en comparación con solo el $24 \%$ en el grupo de hidrocortisona (25). Por otra parte, Frank-Raue y colaboradores, en un ensayo aleatorizado que incluyó a 28 pacientes, compararon la efectividad de la ciproterona frente a la dexametasona y encontraron que en el grupo de ciproterona mejoró el hirsutismo en un 66 \% frente a solo el $31 \%$, res-

Tabla 2. Resultados de laboratorio frente al IMC y la edad*

\begin{tabular}{|c|c|c|c|c|c|c|}
\hline \multirow[t]{2}{*}{ Variable } & \multirow[t]{2}{*}{ Covariable } & \multirow[t]{2}{*}{ Coeficiente } & \multirow[t]{2}{*}{ Valor $\boldsymbol{p}$} & \multicolumn{2}{|c|}{ IC $95 \%$} & \multirow[t]{2}{*}{$R$-squared } \\
\hline & & & & Inferior & Superior & \\
\hline \multirow{3}{*}{$\begin{array}{l}\text { 17-OH } \\
\text { progesterona }\end{array}$} & Edad & 0,1727739 & 0,391 & $-0,231203$ & 0,5767509 & 0,1225 \\
\hline & IMC & $-0,8921113$ & 0,038 & $-1,732771$ & $-0,0514512$ & \\
\hline & Otras & 6,676161 & 0,582 & $-17,72689$ & 31,07921 & \\
\hline \multirow[t]{3}{*}{ Testosterona } & Edad & 1,291334 & 0,219 & $-0,8009552$ & 3,383624 & 0,0440 \\
\hline & IMC & $-0,9088031$ & 0,674 & $-5,262775$ & 3,445169 & \\
\hline & Otras & $-75,14989$ & 0,235 & $-201,5389$ & 51,23912 & \\
\hline \multirow[t]{3}{*}{ DHEA-S } & Edad & 0,4009611 & 0,875 & $-4,754824$ & 5,556746 & 0,0092 \\
\hline & IMC & 2,695185 & 0,613 & $-8,033801$ & 13,42417 & \\
\hline & Otras & $-366,3112$ & 0,022 & $-677,7568$ & $-54,86546$ & \\
\hline
\end{tabular}

*Modelo lineal multivariado, muestra cambios en los niveles de testosterona, 17-OH progesterona y DHEA-S según la edad, el IMC ajustado y el aumento de las dosis de corticosteroides. En relación con la 17-OH progesterona, el nivel está influenciado por el IMC $(p=0,038)$. El cambio de testosterona y DHEA-S depende de su nivel inicial y no está influenciado por otras covariables. 
pectivamente (26). En la presente cohorte, todos los pacientes recibieron ciproterona oral durante un período de 12 meses, ya que el $100 \%$ de los pacientes presentó algún grado de hirsutismo y terapia de reemplazo con dexametasona a dosis ajustadas de acuerdo con la respuesta paraclínica. El resultado fue una disminución significativa en los niveles de la hormona 17-0H progesterona, de 15,9 ng/mL a 1,65 ng/mL a los 6 meses y a $1,1 \mathrm{ng} / \mathrm{mL}$ a los 12 meses de tratamiento.

En varias revisiones se ha evidenciado la influencia del IMC y la edad en los valores de andrógenos femeninos $(27,28)$, por lo que quisimos correlacionar estas variables con cada uno de los paraclínicos evaluados durante el seguimiento (17-0H progesterona, testosterona y DHEA-S), y se encontró que los valores de testosterona y DHEA-S dependen solo de su valor inicial, no están influenciados por la edad o el IMC; mientras que los valores de 17-OH progesterona están influenciados por el IMC. Esto se debe tener en cuenta en pacientes con sobrepeso, cuyo curso de seguimiento bioquímico puede presentar un cambio más lento que en los pacientes con IMC normal.
Dentro de las limitaciones de nuestro estudio están el tamaño reducido de la muestra, la ausencia de un diagnóstico genético y molecular, como lo recomiendan algunas sociedades de endocrinología, debido a limitaciones técnicas y económicas, y el no seguimiento de los cambios clínicos por medio de la escala de Ferriman y Gallwey en cada paciente, el cual no se realizó porque el cambio de dosis de dexametasona se llevó a cabo según los niveles de 17-0H progesterona basal.

Finalmente, dado que es una patología de baja prevalencia sin estudios de seguimiento previos en nuestro país y ante la ausencia de un consenso del régimen de glucocorticoide a utilizar, recomendamos realizar un estudio de casos y controles, y un ensayo clínico, respectivamente.

\section{Agradecimientos}

Los autores agradecen a las instituciones que han hecho posible la realización del trabajo presentado: el Centro Médico Imbanaco y la Universidad Libre de Cali.

\section{Referencias}

1. Bidet M, Bellanné-Chantelot C, Galand-Portier M-B, Tardy V, Billaud L, Laborde K, et al Clinical and molecular characterization of a cohort of 161 unrelated women with nonclassical congenital adrenal hyperplasia due to 21-hydroxylase deficiency and 330 family members. J Clin Endocrinol Metab. 2009;94(5):1570-8.

2. Latorre S, Garzón C, Manosalva G, Merchán S, Jacomussi L, Maldonado S. Hiperplasia adrenal congénita por déficit de 21 hidroxilasa: un reto diagnóstico y terapéutico. Repert Med Cir. 2016;25(2):79-88.

3. Escobar-Morreale HF, Sanchón R, San Millán JL. A prospective study of the prevalence of nonclassical congenital adrenal hyperplasia among women presenting with hyperandrogenic symptoms and signs. J Clin Endocrinol Metab. 2008;93(2):527-33.

4. Parajes S, Loidi L, Reisch N, Dhir V, Rose IT, Hampel R, et al. Functional consequences of seven novel mutations in the CYP11B1 gene: four mutations associated with non classic and three mutations causing classic 11 beta\}hydroxylase deficiency. J Clin Endocrinol Metab. 2010;95(2):779-88.

5. Reisch N, Arlt W, Krone N. Health problems in congenital adrenal hyperplasia due to 21-hydroxylase deficiency. Horm Res Paediatr. 2011;76(2):73-85.

6. El-Maouche D, Arlt W, Merke DP. Congenital adrenal hyperplasia. Lancet. 2017;390(10108):2194-2210.

7. Lin-Su K, Nimkarn S, New MI. Congenital adrenal hyperplasia in adolescents: diagnosis and management. Ann N Y Acad Sci. 2008;1135:95-8.

8. Speiser PW. Nonclassic adrenal hyperplasia. Rev Endocr Metab Disord. 2009;10(1):77-82.

9. New MI. Extensive clinical experience: nonclassical 21-hydroxylase deficiency. J Clin Endocrinol Metab. 2006;91(11):4205-14.

10. Siegel SF, Finegold DN, Urban MD, McVie R, Lee PA. Premature pubarche: etiological heterogeneity. J Clin Endocrinol Metab. 1992;74(2):239-47.

11. Merino P, Bachega T, Céspedes P, Trejo L, Billerbeck AE, Codner E. Utilidad del estudio molecular de CYP21A2 en el manejo prenatal de hiperplasia suprarrenal congénita: detección de dos nuevas mutaciones en Chile. Rev Méd Chile. 2007;135(11):1450-5.

12. Alonso M, Ezquieta B. Hiperplasia suprarrenal congénita no clásica o tardía. Rev Esp Endocrinol Pediatr. 2012; 3(Suppl):61-73.

13. Moran C, Azziz R, Carmina E, Dewailly D, Fruzzetti F, Ibanez L, et al. 21-Hydroxylase-deficient nonclassic adrenal hyperplasia is a progressive disorder: a multicenter study. Am J Obstet Gynecol. 2000;183(6):1468-74.

14. Finkielstain GP, Kim MS, Sinaii N, Nishitani M, Van Ryzin C, Hill SC, et al. Clinical characteristics of a cohort of 244 patients with congenital adrenal hyperplasia. J Clin Endocrinol Metab. 2012;97(12):4429-38.

15. Nimkarn S, Lin-Su K, New MI. Steroid 21 hydroxylase deficiency congenital adrenal hyperplasia. Pediatr Clin North Am. 2011;58(5):1281-300.
16. Loriaux DL. An approach to the patient with hirsutism. J Clin Endocrinol Metab. 2012;97(9):2957-68.

17. Labarta JI, Bello E, Ruiz-Echarri M, Rueda C, Martul P, Mayayo E, et al. Estado en la edad adulta y propuesta de optimización terapéutica de la hiperplasia suprarrenal congénita. An Pediatr 2003;58(Supl 2):12-34.

18. Carvajal Martínez F, Montesino Estévez T, Espinosa Reyes T, Navarrete Cabrera J, Pérez Gesén C. Forma no clásica de hiperplasia adrenal congénita en la niñez y adolescencia. Rev Cubana Endocrinol. 2010;21(1): 62-73.

19. Savaș-Erdeve S, Cetinkaya S, Abalı ZY, Poyrazoğlu S, Baș F, Berberoğlu $\mathrm{M}$, et al. Clinical, biochemical and genetic features with nonclassical 21-hydroxylase deficiency and final height. J Pediatr Endocrinol Metab. 2017;30(7):759-766.

20. Speiser PW. Congenital Adrenal Hyperplasia. F1000Res. 2015;4(F1000 Faculty Rev):601.

21. Carmina E, Dewailly D, Escobar-Morreale HF, Kelestimur F, Moran C, Oberfield S, et al. Non-classic congenital adrenal hyperplasia due to 21-hydroxylase deficiency revisited: an update with a special focus on adolescent and adult women. Hum Reprod Update. 2017;23(5):580-599.

22. Grandone A, Marzuillo P, Luongo C, Toraldo R, Mariani M, Miraglia Del Giudice E, et al. Basal levels of 17-hydroxyprogesterone can distinguish children with isolated precocious pubarche. Pediatr Res. 2018;84(4):533-6.

23. Spritzer P, Billaud L, Thalabard JC, Birman P, Mowszowicz I, Raux-Demay $\mathrm{MC}$, et al. Cyproterone acetate versus hydrocortisone treatment in lateonset adrenal hyperplasia. J Clin Endocrinol Metab. 1990;70(3):642-6.

24. Livadas S, Dracopoulou M, Dastamani A, Sertedaki A, Maniati-Christid M, Magiakou AM, et al. The spectrum of clinical, hormonal and molecular findings in 280 individuals with nonclassical congenital adrenal hyperplasia caused by mutations of the CYP21A2 gene. Clin Endocrinol (Oxf). 2015;82(4):543-9.

25. Young J, Tardy V, de la Perrière AB, Bachelot A, Morel Y; French Society of Endocrinology. Detection and management of late-onset 21-hydroxylase deficiency in women with hyperandrogenism. Ann Endocrinol (Paris). 2010;71(1):14-8.

26. Falhammar H, Nordenström A. Nonclassic congenital adrenal hyperplasia due to 21-hydroxylase deficiency: clinical presentation, diagnosis, treatment, and outcome. Endocrine. 2015;50(1):32-50.

27. Frank-Raue K, Junga G, Raue F, Vecsei P, Ziegler R. Therapie des Hirsutismus bei Frauen mit adrenalen Enzymdefekten der Steroidhormonbiosynthese: Vergleich von Dexamethason mit Cyproteronacetat. Klin Wochenschr. 1990;68(12):597-601.

28. Demers LM. Biochemistry and laboratory measurement of androgens in women. En: Redmond GP (editor). Androgenic disorders. Nueva York: Raven Press; 1995. p. 21-34. 


\section{Hay órganos que sufren y no se ven ${ }^{1}$ La diabetes es más que glucosa ${ }^{1}$}

\section{Uso Temprano}
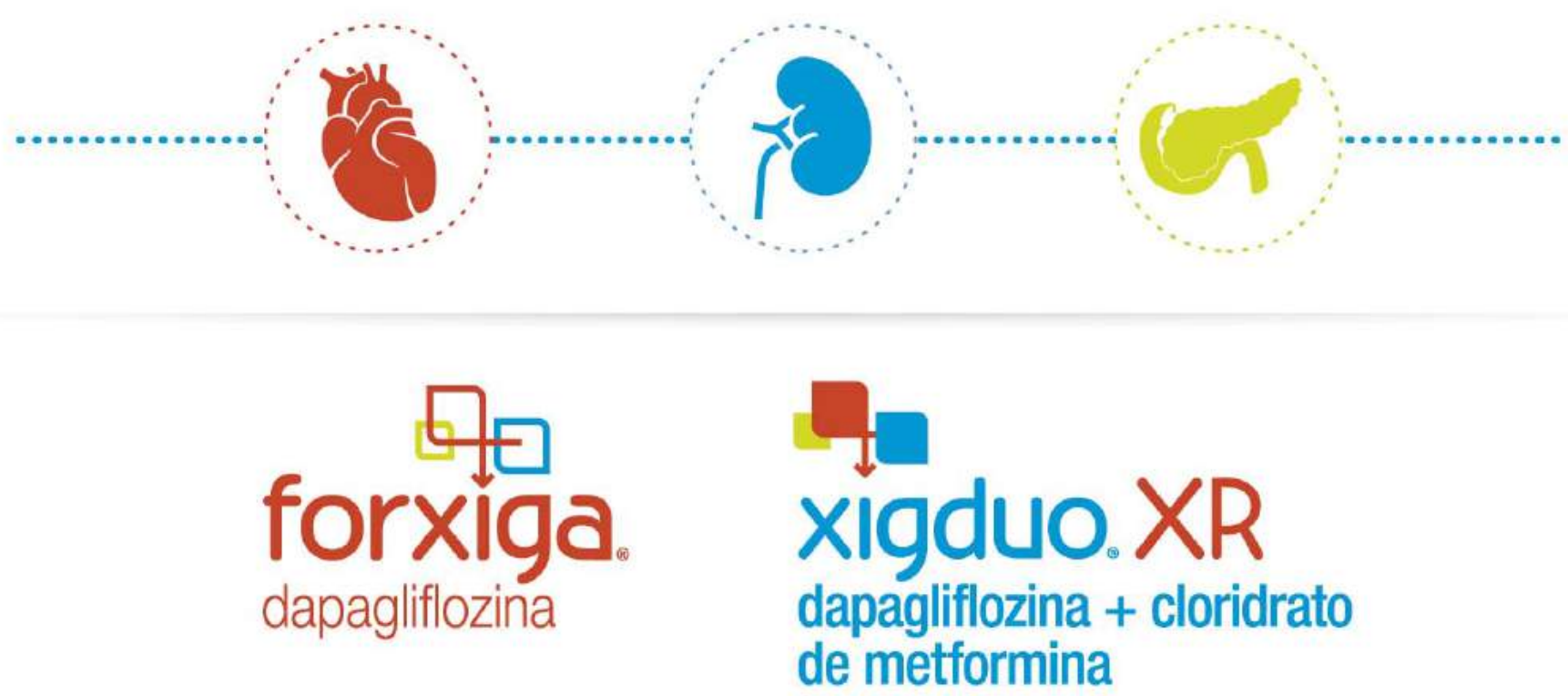

\section{EFICACIA: $\downarrow H b A 1 c$, Peso y PA².8}

\section{AMPLIO PERFIL DE SEGURIDAD ${ }^{2 \cdot 3}$}

\section{CONCIENCIA CARDIORRENAL23,9}

Esto puede ocurrir de forma temprana, incluso antes de que su paciente sea dignosticado ${ }^{10}$ ¿Está su paciente con DMT2 protegido?

HbA1c: hemoglobina glucosilada; PA: Presión Arterial; DMT2: diabetes mellitus tipo 2.

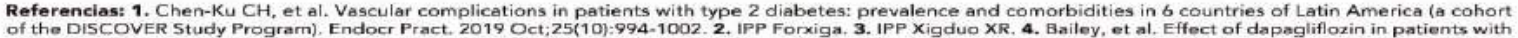
type 2 diabetes who have inadequate glycaemic control with metformin: a randomised, double-blind, placebo-controlled trial. Lancet. 2010;375(9733):2223-2233. Analysis of Dapagliflozin versus Saxagliptin as Add-On Therapy in Patients with Type 2 Diabetes Inadiequately Controlled with Metformin. Presented at the 14th Annual World Congress on Insulin Resistance, Diabotes \& Cardiovascular Disease (WCIRDC), Los Angeles, CA, USA; Supported by: December 1-3, 2016. 8. Rosenstock J et al. Arch Endocrinol Metab 2018; 62(4):424 430. 9. Sonesson, et al. Cardiovascular effects of dapagliflozin in patients with type 2 diabetes and
different risk categories: a meta-analysis. Cardiovase Diabetol (2016) 15:37. 10. Gedebjerg, et al. Prevalence of micro-and macrovascular diabetes complicatians

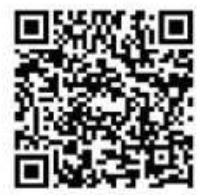

Forxiga" 